\title{
The Diurnal Temperature Range for Europe: A Search for Cosmic Ray Forbush Decrease Manifestations and the DTR Periodicities
}

\author{
A. D. Erlykin ${ }^{1,2}$ and A. W. Wolfendale ${ }^{2}$ \\ ${ }^{1}$ Nuclear Physics and Astrophysics Division, P.N.Lebedev Physical Institute, Moscow 119991, Russia \\ ${ }^{2}$ Department of Physics, Durham University, Durham DH1 3LE, UK
}

Correspondence should be addressed to A. D. Erlykin; erlykin@sci.lebedev.ru

Received 20 September 2012; Accepted 23 October 2012

Academic Editors: G. A. Gerosa and J. M. Vilaplana

Copyright (c) 2013 A. D. Erlykin and A. W. Wolfendale. This is an open access article distributed under the Creative Commons Attribution License, which permits unrestricted use, distribution, and reproduction in any medium, provided the original work is properly cited.

\begin{abstract}
Following on previous work by others, which gave evidence for few days' changes in the European Diurnal Temperature Range (DTR) apparently correlated with Cosmic Ray Forbush Decreases, we have made an independent study. We find no positive evidence. An analysis has also been made of the Fourier components of the time series of the DTR value (taken as deviations from a \pm 10 day running mean). Evidence for a number of interesting periods is found, including one at about 27 days, albeit with a variability with time. The same period of solar irradiance (particularly in the UV) is favoured as the explanation.
\end{abstract}

\section{Introduction}

Uncertainty relating to the relevance of Cosmic Rays (CR) to climate change continues. Although some work (our own included-e.g., Erlykin et al. [1-3]) has cast doubts on the claims of Svensmark and Friis-Christensen [4] and others that there is a causal correlation of Low Cloud Cover (LCC, altitude $<3.2 \mathrm{~km}$, i.e., the "boundary layer") with CR intensity, doubts remain. The supporters draw attention to the results from the CLOUD project [5] which give evidence for an effect of accelerator particles on nucleation rates.

The supporters also draw attention to the Diurnal Temperature Range (DTR) data from European stations where there is an apparent correlation with Forbush Decreases (FD) according to Dragic et al. [6].

The work just referred to is in the spirit of studies by $\mathrm{Lu}$ and Sanche [7], and others, which claimed evidence for CR having an effect on Atmospheric Chlorofluorcarbon Dissociation and Ozone depletion in the Polar atmosphere. It is with the DTR work that we are concerned here.

Although the DTR, which is the day-night temperature difference, is affected by a number of atmospheric parameters (cloud cover, precipitation, changes in agriculture, water vapour feedback, etc.) it is an important quantity to study for two reasons.

(i) It can be measured in a straightforward way.

(ii) It decreased on a global scale from the 1950s onwards, at the time when the mean Global temperature started to increase markedly (Global Warming). DTR has in it therefore a number of (often dependent) climate parameters and these could be, in principle, CR dependent (see [8] for details of DTR for Europe for the range 1950-2005).

FD analyses are useful in that for the short time spans involved (days) phenomena other than CR might be expected to change only slowly so that CR effects dominate.

\section{Previous FD Studies}

Searches for the correlation of FD with other atmospheric parameters have already been made. Specifically, Svensmark et al. [9] searched for, and found, changes correlated with strong FD for the atmospheric liquid water fraction (LCF) - as detected by "MODIS," and Low IR cloud cover 


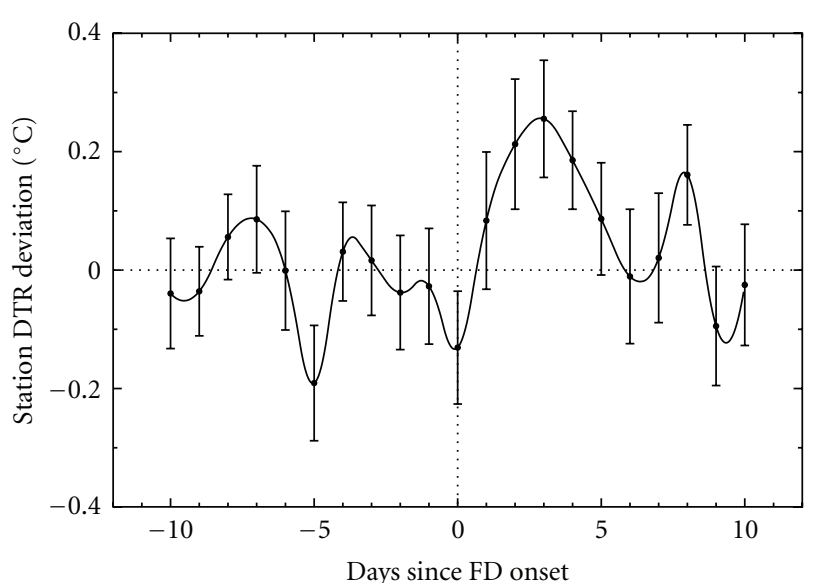

FIgure 1: The results showing the DTR signal following FD decreases. FDs greater than $7 \%$ were selected, of which there were 37. Dragic et al., [6], found a similar, though larger profile.

over regions-as recorded by the "ISCCP" together with aerosol content (using AERONET) and the cloud water content using SSM/1. The results were all similar: the FD was followed within a few days by similar changes in the atmospheric properties.

In the event, Laken et al. [10], concentrating on the LCF (MODIS), found no support for the hypothesis. However, the topic is so important that we have repeated the analysis, but this time using the DTR as the atmospheric parameter under consideration.

This is not the first time such a study has been made. As mentioned already, Dragic et al. [6] found a significant correlation for a limited class of FD: those above $7 \%$ in magnitude. The DTR data were taken from 189 European meteorological stations; the stations were randomly selected but covered the entire European region. Although details will be given later, the "result" can be mentioned here: an increase in mean DTR for FD $>7 \%$ of $(0.38 \pm 0.06)^{\circ} \mathrm{C}$. Taken at its face value the result is very significant.

Although the results used are only for European stations and are thus not Global in any sense, they may, nevertheless, be very useful. As Lockwood [11] remarks in his comprehensive review of solar influences on Global and Regional climates, the latter are much more significant than the former.

\section{The Present Analysis of FD versus DTR}

3.1. Sources of the Data. The data, comprising the daily sequence of mean DTR and CR intensity, were provided by Dragic et al. [6] and Laken (2012, private communication).

3.2. Processing of the Data. There seems to be no contention as to their validity and their manipulation in that our own work confirmed that of Dragic et al. [6], at least in a general way. Figure 1 gives the result from our own analysis for FD $>7 \%$. It should be mentioned that the amplitude of the effect presented in Figure 1 is smaller and the uncertainties, errors, presented in Figure 1 are bigger than those given by

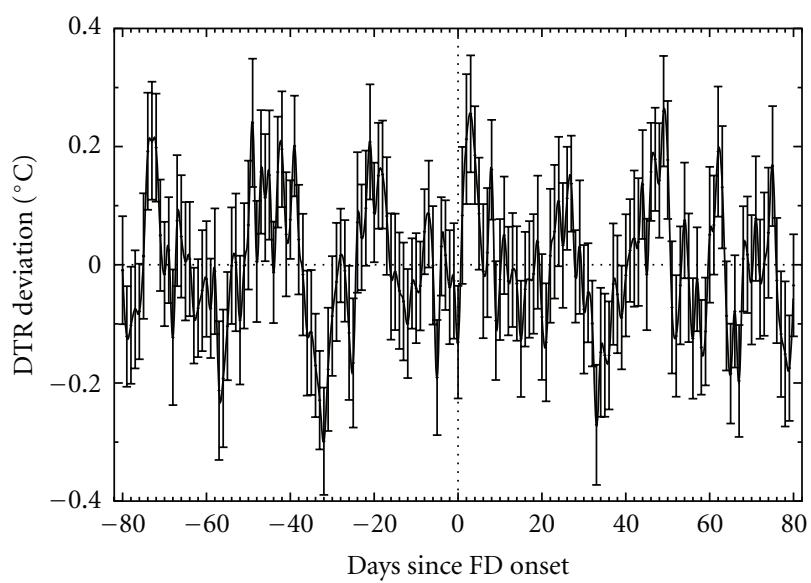

FIgURE 2: Profile of mean DTR deviations from the 21-day running mean averaged over \pm 80 days around the onset of 37 FD with magnitude $>7 \%$.

Dragic et al. [6], namely, $0.25 \pm 0.10^{\circ} \mathrm{C}$, to be compared with $0.38 \pm 0.06^{\circ} \mathrm{C}$ (see Section 2 ); this topic is taken up again later. The argument is the extent to which the profile can be regarded as significant.

3.3. Check on the Chance of Significance. Figure 2 gives the result of our analysis for the 37 events with $\mathrm{FD}>7 \%$; this relates to a wider time range than covered by Figure 1 (i.e., \pm 80 days, to be compared with \pm 10 days). For \pm 10 days the plots coincide precisely with the plot given in Figure 1. It is immediately apparent that peaks similar to that for \pm 10 days are not infrequent and, furthermore, the difference between pre- and post-FD is not significant. The problem is to determine the frequency of a peak which would be counted as acceptable, that is, would be evidence for a (strong) FD causing a significant increase in the DTR. There are two parameters to be considered, as follows.

(1) The probability of a peak of acceptable height, and time width.

(2) The phase lag between the FD onset and the onset of the DTR increase. In [6] it was zero days for FD $>7 \%$, +1 day for FD: $7-10 \%$, and -1 day for FD $>10 \%$. In [9] it was approximately +6 days.

Starting with (1) we have smoothed the DTR data with a 21-day running mean and studied the patterns following datum times where the deviation from the datum level, $\Delta$ (DTR), was less than $0.05^{\circ} \mathrm{C}$. We consider that 2 to 4 consecutive displacements above $0.2^{\circ} \mathrm{C}$ would give patterns as acceptable evidence for a significant DTR signal (see Figures 1 and 2). The results are given in Table 1.

It is seen that a probability of about $15 \%$ is indicated for zero time delay (phase lag).

Concerning the phase lag that would have been regarded as allowable, this appears to be at least 3 days. Thus, the overall probability of a chance pattern being accepted as genuine is about $45 \%$. 
TABLE 1: Analysis of the chance probability of 2 (or 4 ) consecutive values of the DTR, $\Delta$, being greater than $0.2^{\circ} \mathrm{C}$ ( or $0.1^{\circ} \mathrm{C}$ ), denoted $\Delta 2,0.2^{\circ} \mathrm{C} ; \Delta 2,0.1^{\circ} \mathrm{C}, \Delta 4,\left(0.2^{\circ} \mathrm{C}\right), \Delta 4,\left(0.1^{\circ} \mathrm{C}\right)$. Values for negative excursions are also indicated; they are essentially the same as for the positive excursions.

\begin{tabular}{lccc}
\hline$\Delta 2,0.2^{\circ} \mathrm{C}$ & $20 \%$ & $\Delta 4,0.2^{\circ} \mathrm{C}$ & $8 \%$ \\
$\Delta 2,0.1^{\circ} \mathrm{C}$ & $26 \%$ & $\Delta 4,0.1^{\circ} \mathrm{C}$ & $12 \%$ \\
\hline$\Delta 2,-0.2^{\circ} \mathrm{C}$ & $22 \%$ & $\Delta 4,-0.2^{\circ} \mathrm{C}$ & $9 \%$ \\
$\Delta 2,-0.1^{\circ} \mathrm{C}$ & $29 \%$ & $\Delta 4,-0.1^{\circ} \mathrm{C}$ & $10 \%$ \\
& $( \pm 2 \%)$ & & $( \pm 1 \%)$ \\
\hline
\end{tabular}

TABLE 2: The two most significant peaks in the Fourier plots for different time ranges.

\begin{tabular}{lcc}
\hline Time range & & \\
\hline 80 days before FD & $25(\mathrm{~B})$ & 11 \\
80 days after FD & $22(\mathrm{~B})$ & 12 \\
(graphs not shown) & & $11,13(\mathrm{~B})$ \\
13,673 days & 28,30 & \\
(the whole period) & $22(\mathrm{~B}) 27$ & $13(\mathrm{~B})$ \\
Cycle 21 & 30 & \\
Cycle 22 & $28(\mathrm{~B}) 27$ \\
Cycle 23 &
\end{tabular}

"B" denotes the bigger of the two. For close pairs both are given.

Moving on from Forbush Decreases we examine the periodicity in the DTR deviations; Figure 2 leads us to believe that there are one or more such periodicities.

\section{The Periodicity of DTR Excursions}

4.1. The Analysis. The presence of a periodicity (or periodicities) could have relevance to solar effects, or related to CR effects. Periodicities in the region of 27 days might be expected in that this is the (mean) solar rotation period although it is appreciated that the rotation period varies from about 25 days at the Equator to 38 days at the Poles and a clear result may not be expected.

In the whole data set some $1.37 \times 10^{4}$ continuous daily values of the DTR deviations (from the 21 day running mean) are available. A Fourier analysis yielded the results shown in Figure 3.

The expected peaks at 12 months and 6 months are clearly visible, thus confirming the quality of the data. Other significant sharp peaks are present at the harmonics of the annual peak, namely, one year divided by 3,4, and so forth. Physically interesting peaks are also present near 27 days and 13 days. In Table 2 we give the periods for the two most significant peaks in the profiles, for various data sets, as follows.

(i) 80 days before the FD events and 80 days afterwards (Figure 2; the amplitudes of the Fourier transforms are not given).

(ii) The whole period of observation (Figure 3).

(iii) Each solar cycle $(21,22$, and 23) separately.

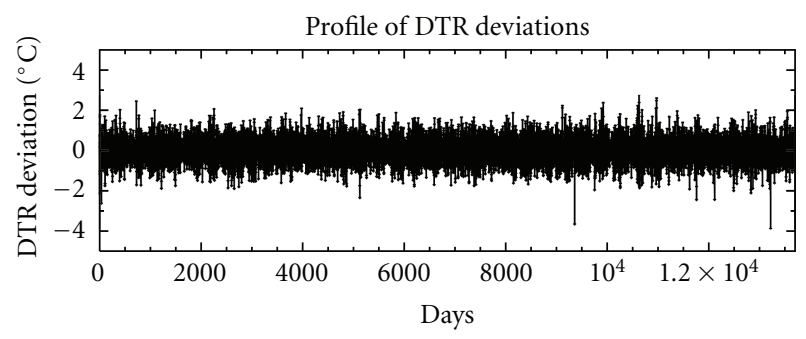

(a)

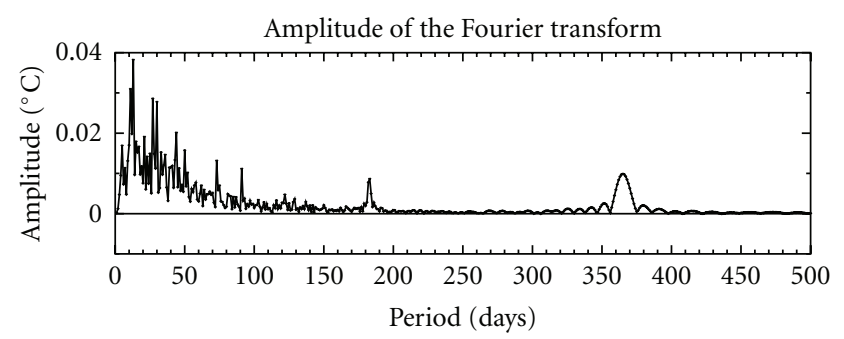

(b)

FIgURE 3: Profile of DTR deviations from the 21-day running mean (as for Figure 2) for the whole period of 13,673 days, together with the amplitude of the Fourier transforms.

It is evident from Table 2 that there is some grouping of the periods round $27 \mathrm{~d}$ (actually $22 \mathrm{~d}$ to $30 \mathrm{~d}$ ) and $13 \mathrm{~d}$ (actually $11 \mathrm{~d}$ and $13 \mathrm{~d}$ ). To say that we have confirmed these periods would be too strong a statement but there is some evidence favouring them. In what follows an examination is given accepting the evidence as real.

4.2. Interpretation of the Possible $27 d$ and $13 d$ Peaks. A 27day peak is interesting in that it is clearly solar related, 27day being the mean period of solar rotation, as remarked in Section 4.1. It is well known that there is a near 27-day CR intensity variation (e.g., [12]) for which the amplitude is about $0.4 \%$ [13]. There is also a difference between alternate solar cycles. These features are attributed to energetic processes on the surface of the sun which give rise to timedependent solar winds, and thereby periodicities of about 27 and 13 days, the latter arising from frequent $180^{\circ}$ longitude differences in the solar surface disturbances [14].

There is a problem with the solar-related periodicity analysis, however, due to the well-known fact that the solar rotation period is solar-latitude related. Thus, our analysis of different lengths of time may give different periods. This topic has been followed up by examining the results cycle by cycle, namely, for solar cycles 21,22 , and 23.

This is the reason for giving the division shown in Figure 4 for which details are given in Table 2. The results are rather disappointing in that there is no equality in the patterns for the alternate cycles: 21 and 23. Nevertheless, we maintain that there is some modest evidence favouring identification of solar-rotation features in the DTR record.

4.3. The Quoted Errors in the DTR Measurements. A good feature of Figure 2, which was used for periodicity studies, is 


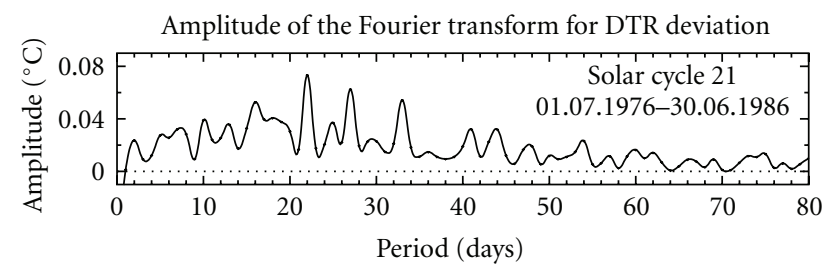

(a)

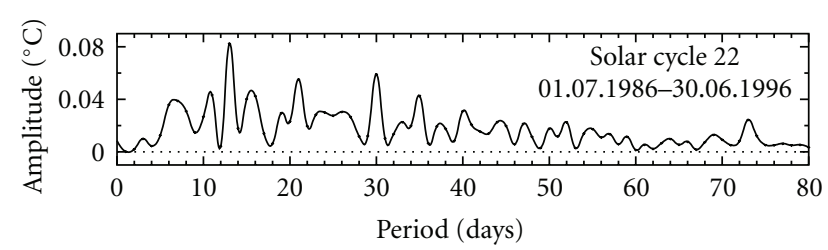

(b)

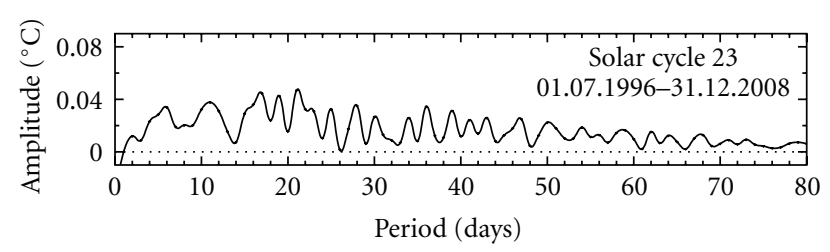

(c)

FIGURE 4: Profiles of Fourier amplitudes for the solar cycles 21, 22, and 23 .

that it allows a check on the veracity of our quoted errors. The numbers of (daily) values greater than 0,1 , and 2 standard deviations are 360, 106, and 11. For a Gaussian we expect 360, 120 , and 18. It follows that, due to the data being deviations from a running mean, our "errors" are slightly overestimated. Using the numbers just given the overestimate is calculated to be by $10 \%$. This is much smaller than the $70 \%$ by which our errors exceeded those of Dragic et al. [6] in their important paper which stimulated the present work. The origin of the difference is not known.

\section{The DTR-Cosmic Ray Correlation: Is It Causal?}

There is the standard problem of whether in the DTR the possible 13 and 27 day periods are Cosmic Ray related (i.e., there is a causal connection), or whether both variations are caused by a third phenomenon. The alternatives here are therefore that the DTR periodicity is caused by (i) solar luminosity per se, which could cause climate change, or (ii) cosmic rays, via ionization affecting the degree of cloud cover.

It appears that no analysis of the results, alone, can make a distinction, because the Total Solar Irradiation (TSI) and Sunspot number are tightly correlated for obvious reasons and the Sunspot number and CR intensity are correlated by way of the variation in the Solar Wind.

Instead, recourse must be made to other results.
We consider that solar irradiance variations are a more likely source of the DTR periods than CR for the following reasons.

(i) The source of the DTR change itself is claimed to be "changes in (terrestrial) emissions and the associated changes in incoming solar radiation" [8]. Thus, changes in the solar irradiance are preferred. In this context, Rotman [15] has drawn attention to the very large amplitude of the 27-day variation of the UV (120-300 nm) irradiance.

(ii) The CR ionization mechanism runs into serious difficulties. Although it is true that for Cycle 22 the CR Low Cloud Cover (LCC) correlation was strong [4] that for the next cycle was not (see, e.g., [1]). Most importantly, the last-mentioned authors drew attention to the fact that the CR and MCC are anticorrelated to the extent that the sum of LCC and MCC (and indeed the total CC) is not correlated with the CR. Thus, the role of CC in causing the groundlevel irradiance variation which contributes to the DTR, CR correlation (at least for an 11-year period) is negligible.

Other serious problems for CR contributing via ionization to Cloud Cover come from the following.

(i) Ionization caused by nuclear explosions, terrestrial radon, and the Chernobyl nuclear accident had no effect on Cloud Cover [2].

(ii) Those clouds (stratiform), which should have a greater sensitivity to CR, do not show it [3].

(iii) Recent work at CLOUD [5] has shown that ionizing particles do produce nucleation under carefully controlled conditions, at least using the atmospherically relevant $\mathrm{H}_{2} \mathrm{SO}_{4}$. However, there is a very great temperature dependence of the rate and for most of the troposphere, and particularly the "boundary layer"-at altitudes below several $\mathrm{km}$-the atmospheric temperatures are too high. Kirkby et al. agree with this diagnosis [5].

The causal nature of the tropospheric CR, CC correlation on the 11-year scale and the DTR correlation on the four-day (Forbush Decrease), 27-day, and 1-year scales is thus strongly disfavoured. An explanation in terms of changes to the solar irradiance is more likely.

The dismissal of a causal connection for tropospheric climate parameters and CR does not mean that CRs have no effect on the terrestrial atmosphere as a whole. There is evidence for CR effects in the stratosphere as mentioned in Section 1 . These are very small, however, and unlikely to be of much relevance to the lower atmosphere, changes in which are of such contemporary concern.

Finally, it should be noted that the slow increase in the CR intensity over the past two decades, as evinced by neutron monitors (e.g., that as on the Oulu web site [16]), shows that even if there was a CR, tropospheric atmosphere link it would not contribute to the observed current Climate Change. 


\section{Conclusions}

Concerning the effect of Forbush Decreases on the Diurnal Temperature Range, we find no evidence which supports the conclusions of Dragic et al. [6]. We estimate that there is a $45 \%$ chance of an "interesting" and acceptable DTR pattern following a strong FD by statistical fluctuations alone. A Fourier analysis of the DTR pattern ("DTR" being, as before, a deviation from $\mathrm{a} \pm 10$ day running mean) confirms the quality of the data.

The periods for the DTR data detected in the Fourier study are of interest in their own right. The periods are consistent with those exhibited by both solar irradiance and CR. Other evidence disfavouring CR connections indicates that solar irradiance changes are presumably responsible.

Different arguments, too, contribute to the conclusion that CRs have a negligible effect on the tropospheric climate.

\section{References}

[1] A. D. Erlykin, "An emission layer as a gravity wave detector," Journal of Atmospheric and Solar-Terrestrial Physics, vol. 71, no. 17-18, pp. 1974-1981, 2009.

[2] A. D. Erlykin, G. Gyalaic, K. Kudelac, T. Sloand, and A. W. Wolfendaleb, "Some aspects of ionization and the cloud cover, cosmic ray correlation problem," Journal of Atmospheric and Solar-Terrestrial Physics, vol. 71, no. 8-9, pp. 823-829, 2009.

[3] A. D. Erlykin, T. Sloanc, and A. W. Wolfendaleb, "The search for cosmic ray effects on clouds," Journal of Atmospheric and SolarTerrestrial Physics, vol. 71, no. 8-9, pp. 955-958, 2009.

[4] H. Svensmark and E. Friis-Christensen, "Variation of cosmic ray flux and global cloud coverage-a missing link in solar-climate relationships," Journal of Atmospheric and SolarTerrestrial Physics, vol. 59, no. 11, pp. 1225-1232, 1997.

[5] J. Kirkby, J. Curtius, J. Almeida et al., "Role of sulphuric acid, ammonia and galactic cosmic rays in atmospheric aerosol nucleation," Nature, vol. 476, pp. 429-433, 2011.

[6] A. Dragic, I. Aničin, R. Banjanac et al., "Forbush decreases-clouds relation in the neutron monitor era," Astrophysics and Space Sciences Transactions, vol. 7, pp. 315-318, 2011.

[7] Q. B. Lu and L. Sanche, "Effects of cosmic rays on atmospheric chlorofluorocarbon dissociation and ozone depletion," Physical Review Letters, vol. 87, no. 7, Article ID 078501, 4 pages, 2001.

[8] K. Makowski, M. Wild, and A. Ohmura, "Diurnal temperature range over Europe between 1950 and 2005," Atmospheric Chemistry and Physics, vol. 8, pp. 6483-6498, 2008.

[9] H. Svensmark, T. Bandoll, and J. Svensmark, "Cosmic ray decreases affect atmospheric aerosols and clouds," Geophysical Research Letters, vol. 36, Article ID L15101, 4 pages, 2009.

[10] B. Laken, A. Wolfendale, and D. Kniveton, "Cosmic ray decreases and changes in the liquid water cloud fraction over the oceans," Geophysical Research Letters, vol. 36, Article ID L23803, 2009.

[11] M. Lockwood, "Solar influence on global and regional climates," Surveys in Geophysics, vol. 3-4, pp. 503-534, 2012.

[12] L. Dorman, Cosmic Rays, North Holland, Amsterdam, The Netherlands, 1974.

[13] A. Gil, R. Modzelewska, and M. V. Alania, "Features of the 27-day variations of the galactic cosmic ray intensity and anisotropy," Acta Physica Polonica B, vol. 39, no. 5, p. 1301, 2008.
[14] J. Pap, W. K. Tobiska, and S. D. Bouwer, "Periodicities of solar irradiance and solar activity indices, I," Solar Physics, vol. 129, no. 1, pp. 165-189, 1990.

[15] G. J. Rotman, "27-Day variations observed solar u.v. (120$300 \mathrm{~nm}$ ) irradiance," Planetary and Space Science, vol. 31, no. 9, pp. 1001-1007, 1983.

[16] Oulu Neutron Monitor, http://cr0.izmiran.rssi.ru/oulu/main. htm. 

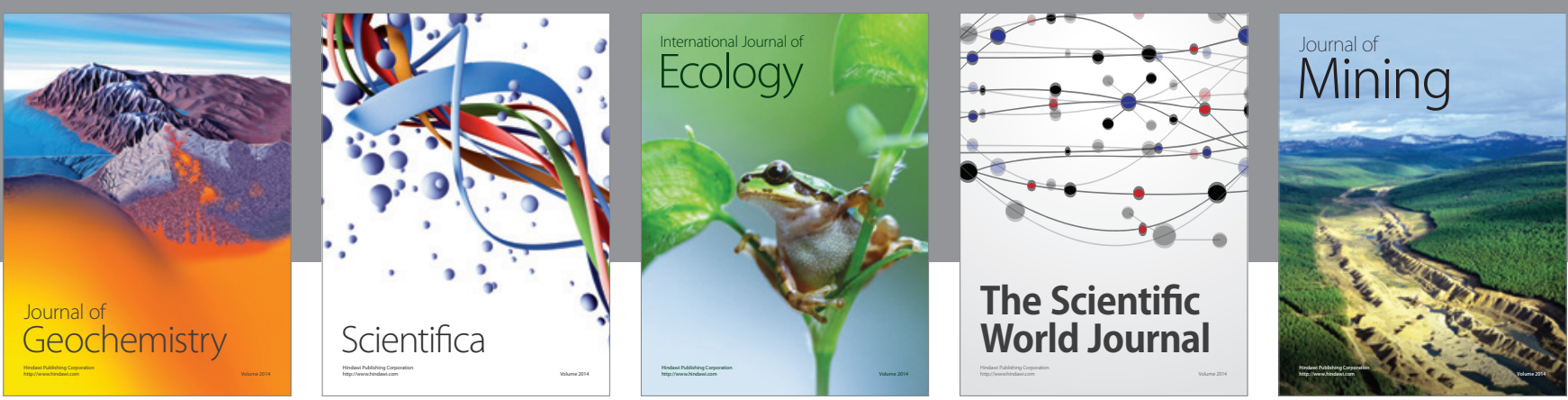

The Scientific World Journal
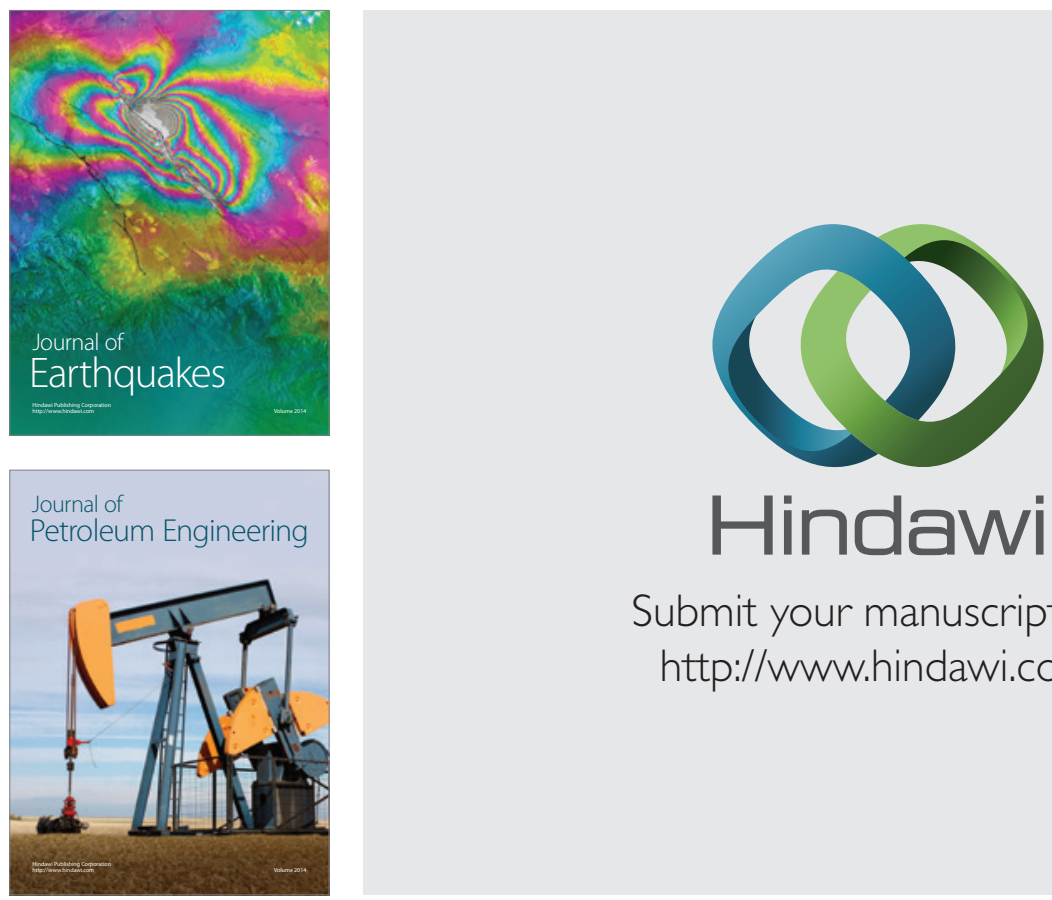

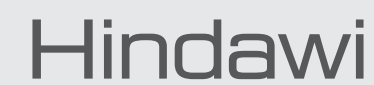

Submit your manuscripts at

http://www.hindawi.com
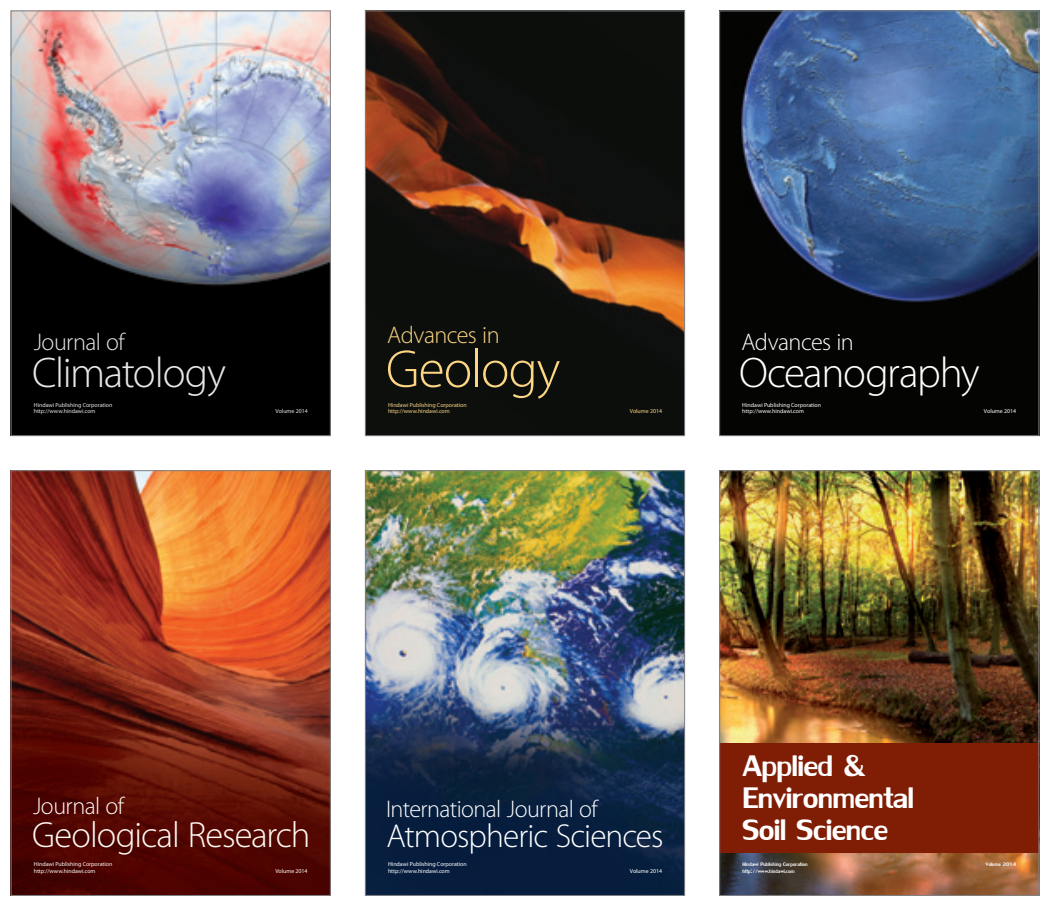
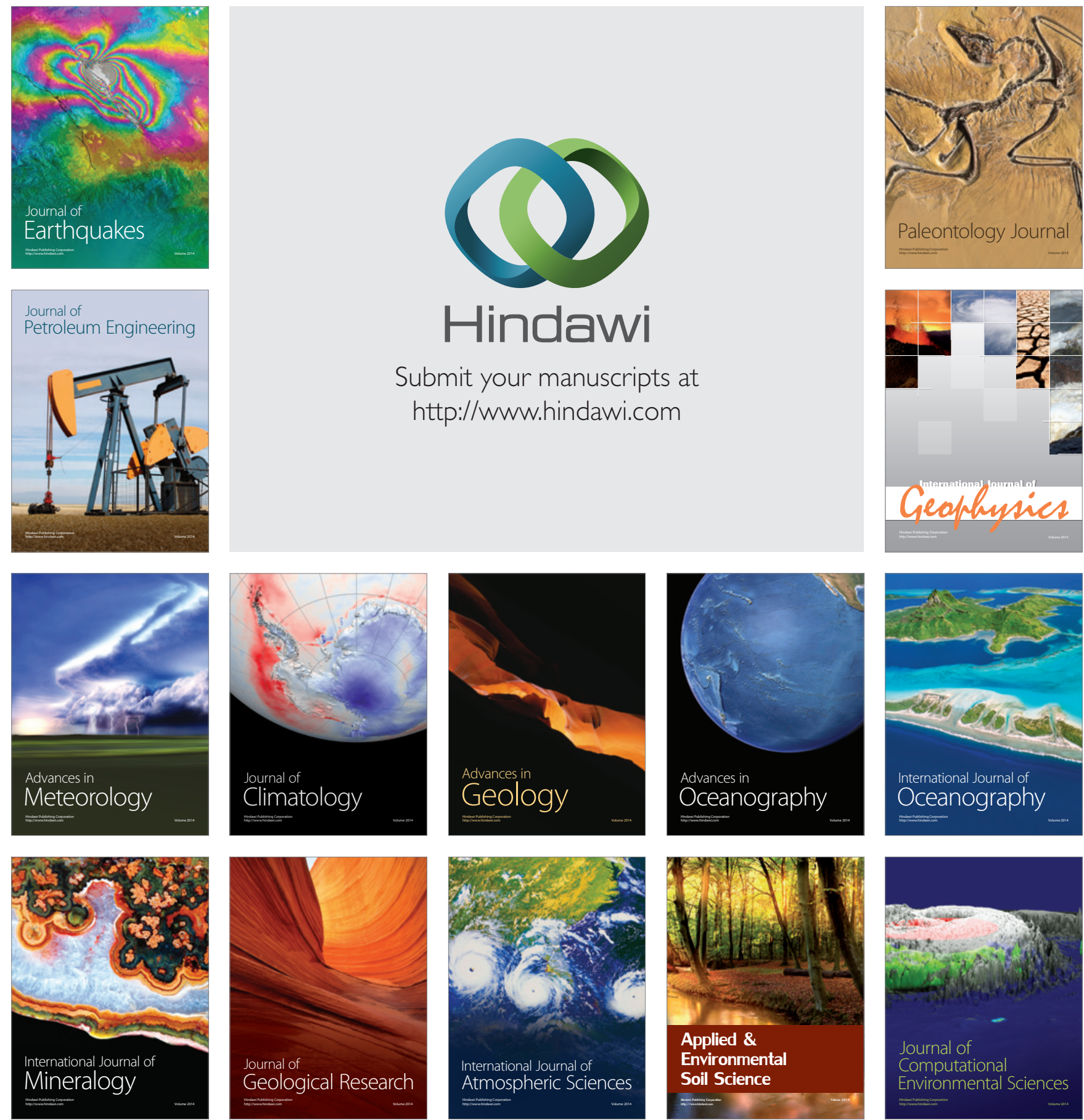